\title{
TWO COORDINATE SYSTEMS DESCRIPTION OF \\ VISCOUS FLOW PAST A CIRCULAR CYLINDER
}

F.P.H. van Beckm

Dept. of Applied Mathematics

Twente University of Technology

Enschede, Netherlands

\section{Summary}

Two dimensional steady viscous exterior flow is often treated as an elliptic problem within a finite region, bounded by a big circle. Solutions by means of Fourier expansions or discretisation on a polar grid will only give reliable results for low Reynolds numbers. In the present paper an example of a transformation is given, that maps the entire vorticity field onto a rectangle so that: (a) the vorticity equation can develop its parabolic character, (b) the wake in the far field comes out properly, and (c) the restriction to low Reynolds numbers is removed. For the elliptic streamfunction equation the polar system is retained.

This two coordinate systems description has been studied for a linearized version of the Navier-Stokes equations obtained by assuming a fixed velocity field $\underline{v}^{(0)}$ in the convection term. The first results $\zeta$ and $\psi$ are qualitatively so similar to the final solutions known from literature, that an iteration process, suggested by deriving a new velocity field $\underline{v}^{(1)}$ from $\psi$, can be expected to show rapid convergence.

2. Mathematical formulation of the problem

For steady two dimensional viscous flow the Navier-Stokes equations read:

$$
\begin{aligned}
& \Delta \zeta-\frac{1}{2} R \underline{V} \cdot \nabla \zeta=0 \\
& \Delta \psi=-\zeta
\end{aligned}
$$

where $\zeta$ is vorticity, $\underline{v}$ velocity, $\psi$ streamfunction $(\operatorname{rot}(0,0, \psi)=\underline{v})$, and the Reynolds number $R$ is based on the diameter. 
For flow past a circular cylinder the boundary conditions, written in polar coordinates $r$ and $\theta$, are:

symmetry: $\theta=0, \pi: \quad \zeta=0, \quad \psi=0$

infinity: $r \rightarrow \infty: \quad \zeta \rightarrow 0, \psi \rightarrow r \sin \theta$

cylinder: $\quad r=1: \quad \psi=0$ and $\frac{\partial \psi}{\partial r}=0$

\section{Two couplings}

We first notice two apparent features in this formulation of the problem. a. Coupling in the partial differential equations: the vorticity is the right-hand side of the streamfunction equation and the streamfunction is occuring - through its derivatives - in the convection term of the vorticity equation. The latter coupling makes the system even nonlinear.

b. Coupling in the boundary conditions: even if the coupling in the partial differential equations would be absent, we still should not be able to solve the vorticity or the streamfunction problem separately because both are ill-posed: the vorticity problem lacks one boundary condition while the streamfunction problem has two.

\section{Restriction relation for polar coordinates}

As an analytic solution is out of the question the problem is taken up by numerical specialists who seem to be challenged mainly by the couplings mentioned in the previous section. Unfortunately the polar coordinate system that often is used in these calculations, is unfit to describe the vorticity in the far field, as can easily be seen from the asymptotic behaviour

$$
\zeta \simeq \frac{Q e^{-\frac{1}{2} Q^{2}}}{r}, \quad Q^{2}=\operatorname{Rr} \sin ^{2} \frac{1}{2} \theta
$$

A picture of $\zeta$ vs $Q$ shows that the main

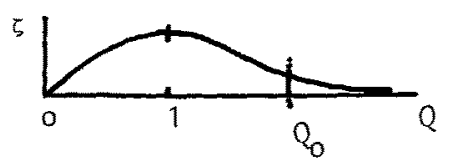
part of the wake is over at about $Q_{0}=2$. The corresponding value of $\theta$ is $\theta_{0}=\frac{4}{\sqrt{\mathrm{Rr}}}$. With $r$ fixed the function $\zeta(x, \theta)$ is practically zero for $\theta>\theta_{0}$. For such a function the dominating coefficients in the expansion $\Sigma b_{n}(r) \sin n \theta$ are those with index $n$ in the neighbourhood of $\pi / \theta_{0}$. So when a separation of variables technique is used with $N$ Fourier components, the reliability of the representation of the vorticity will be restricted by the relation $N^{2}=\frac{\pi^{2}}{\theta_{0}^{2}}=\frac{\pi^{2}}{16^{2}} \mathrm{Rx}$.

In discretisation methods using a polar grid we meet the same relation when we think of $\theta_{0}$ as the angle of the first radial line: if $N$ is the number of radial lines, then $\theta_{0}=\pi / N$. So for $r$ satisfying the above relation the wake is almost confined within the first grid mesh, and the description of the wake further downstream will be very poor. 


\section{Creation of new difficulties}

As the polar system has certain advantages concerning geometry, orthogonality and the ease of progranming, one might accept the restriction relation and achieve accurate results for at least low Reynolds numbers, if there were no other problems.

However, bounding the calculational domain by a big circle one rouses new difficulties, that will be mentioned here only briefly. First, one has the problem which boundary conditions should be imposed on the outer boundary; second, one will be tempted to solve this problem as an elliptic one, thus ignoring the parabolic character the vorticity has downstream; and third, in case the parameters are such that the parabolic character is showing up within the calculational domain, the vorticity will not be inclined to satisfy outer boundary conditions unless by an unnatural change of behaviour.

6. New coordinates for the vorticity equation

In order to remove the restriction relation and to take into account the elliptic character of the vorticity near the cylinder and the parabolic character downstream we search for a coordinate system in which radial lines start from the cylinder as they do in the polar system, but gradually bend over to the wake to coincide asymptotically with curves $\mathrm{Q}=$ constant. An example of an orthogonal system covering these properties is

$$
\begin{aligned}
& \overline{\mathrm{r}} \mathrm{e}^{-\sin ^{2} \frac{1}{2} \bar{\theta}}=a+b \frac{s}{1-s}, \quad 0<s<1 \\
& \bar{r} \tan ^{2} \frac{1}{2} \bar{\theta}=\tan ^{2} \frac{1}{2} \pi t, \quad 0<t<1
\end{aligned}
$$

where $\vec{r}$ and $\bar{\theta}$ are polar coordinates with respect to an origin $O$ on the positive $x$-axis, $a$ is a constant and $b$ is a parameter

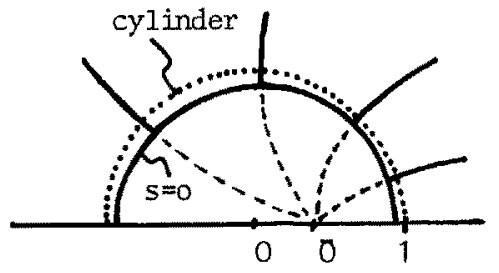
that controls the distribution of grid points. Notice that the entire flow region is mapped onto the unit circle in the $s, t-p l a n e$.

The only point with this transformation is that the curves seconstant are no exact circles. So we cannot do better than to choose the parameters 00 and a such that the curve $s=0$ lies inside the cylinder and as close as possible (maximu deviation about $1 \frac{1}{2}:$ of the radius). When we want to know about $\zeta$ at the cylinder itself, we have to interpolate, but as we retain the polar system for the $\psi$-problem, we have to do a lot of interpolation anyway, so this is not a serious drawback. With respect to our first aim, i.e. to allow of a proper description of the vorticity in the middle and far field, the transformation is satisfactory as will be shown in the following sections.

To test the usefulness of the transformation we solve a linearized version of the Navier-Stokes equations, i.e. we take $\underline{v}$ in the convection term a given velocity field that coincides with the free stream velocity at infinity. In fact we have taken 


$$
\begin{aligned}
& \underline{v}=\underline{0} \text { for } r<r^{*} \\
& \underline{v}=\underline{v}^{\infty} \text { for } r>r^{*} \quad\left(v_{x}^{\infty}=1, v_{y}^{\infty}=0\right)
\end{aligned}
$$

with $r^{*}$ obtained from a rough boundary layer consideration.

\section{Solution of the linearized problem}

a. As the new coordinate system is concentrated in the wake we shall not use it for the $\psi$-problem, because in a two dimensional Poisson equation the forcing term, although of local character, may have a wide influence. We retain essentially the polar system.

We exchange the inhomogeneous condition at infinity for an inhomogeneous boundary condition at the cylinder by introducing

$$
\hat{\psi}=\psi-\left(\mathrm{r}+\frac{1}{\mathrm{r}}\right) \sin \theta
$$

This $\hat{\psi}$ satisfies the well-posed problem $\Delta \hat{\psi}=-\zeta$ with boundary conditions:

$$
\hat{\psi}(\mathrm{r}, 0)=0=\hat{\phi}(\mathrm{r}, \pi), \quad \frac{\partial \hat{\psi}}{\partial \mathrm{r}}(1, \theta)=0, \hat{\psi} \rightarrow 0 \text { for } \mathrm{r} \rightarrow \infty \text {. }
$$

b. We notice that we can solve the vorticity problem as soon as a boundary condition $\zeta(s=0)$ has been given. Next we can solve the $\hat{\psi}$-problem formulated above. In particular we are interested in the resulting $\phi(r=1)$, that has a linear relationship with $\zeta(\mathrm{s}=0)$ because both partial differential equations are linear and all the remaining boundary conditions are homogeneous. So we can get an approximate solution to the linearized problem by solving the $\zeta$ - and the $\psi$-problem several times starting with linearly independent functions $\zeta_{k}(s=0)$, and requiring a linear combination of the resulting functions $\widehat{\psi}_{k}(r=1)$ to be in some sense a good approximation of $-2 \sin \theta$, which corresponds to $\psi(r=1)=1$, the only condition that has been left out till now.

In fact we have taken $\zeta_{k}(s=0)=\sin k \pi t, k=1, \ldots 5$.

c. The $\zeta$-equation is discretized by central differences for the viscous term and upstream differences for the convection term. In a calculational molecule the coupling with the downstrean grid point vanishes as the molecule moves towards infinity $(s=1)$, thus showing the resemblance to an implicit discretisation of a parabolic equation.

For the $\psi$-problem we first map the entire flow field onto the rectangle $0<p<1$, $0<\theta<\pi$, where $\rho$ is defined by

$$
r=1+b^{\prime} \frac{\rho}{1-0}
$$

This transformation is suggested by the asymptotic behaviour $\hat{\psi} \simeq \frac{1}{\mathrm{r}}$.

The discretisation is done by central differencing on an equally spaced grid in the $\rho, \theta$-rectangle. Also in the Neumam condition at the cylinder $(\rho=0)$ central differences are used. 


\section{Results and discussion}

The algorithm described in sections 6 and 7 has been run for $R=100$. The behaviour of the vorticity shown in fig. 1 is in good accordance with the asymptotic expression given in section 4: for fixed $s$ the shape is similar to the $Q-\zeta$ picture, and for $s \rightarrow 1$ the whole vorticity distribution is decreasing linearly with $s$ which corresponds with the factor $\frac{1}{\mathrm{r}}$ in the asymptotic expression.

Fig. 1 The wake near infinity as a function of $s$ and $t$, for $R=100$ calculated with a $16 \times 16 \mathrm{grid}$

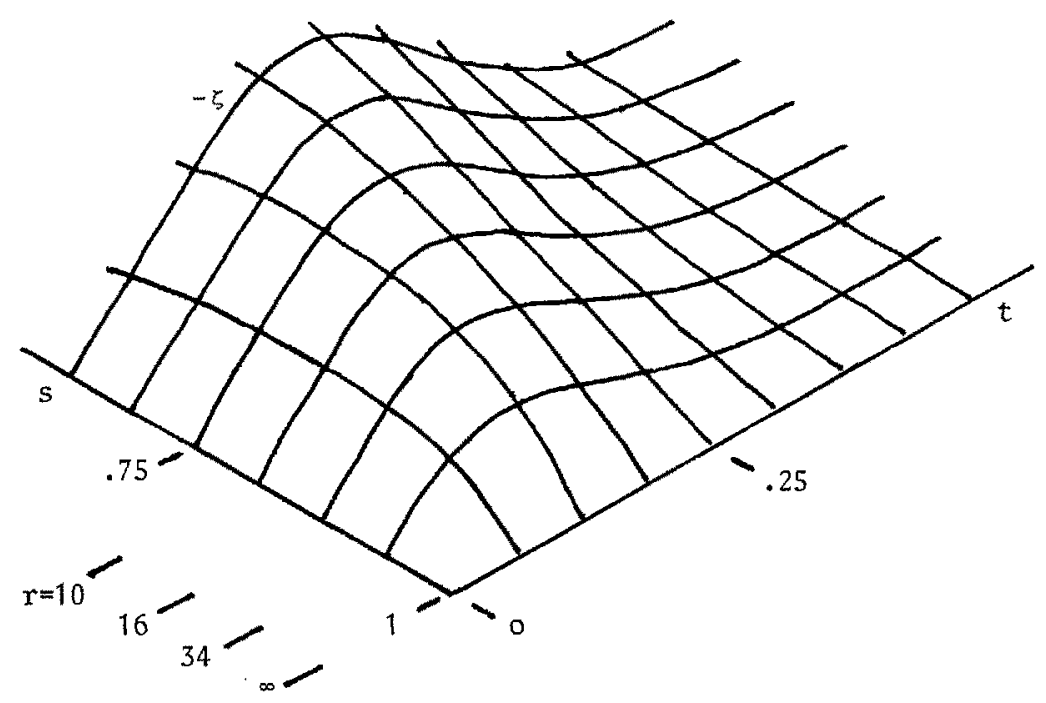

A picture of the vorticity in the fysical plane is given in fig.2. It was calculated for $\mathrm{R}=20$ in order to have some comparison with literature. Of course numerical agreement is out of the question because we only have solved the linearized equations; but some qualitative aspects are encouraging e.g. the shape of the curves of constant vorticity, the location of maximm vorticity at the cylinder, and negative vorticity values for small values of $\theta$. (The last two features could not be represented in the picture.)

The streamfunction picture (fig, 3 ) is qualitatively still better. Recirculation is showing, and even the size of the recirculation area is in reasonable agreement with solutions of the full Navier-Stokes equations known from literature. We may say that the velocity field derived from this streamfunction solution will show a substantial improvement of the first guess for $\underline{v}$ (see section 6 ). So we would think that repeating the whole procedure with this new $\underline{v}$ could be the first step of a 
rather fast converging iteration process.

Fig. 2 Vorticity distribution for $R=20, r^{*}=1.8$, calculated with a $10 \times 20$ grid $(\cdot=$ grid point $)$

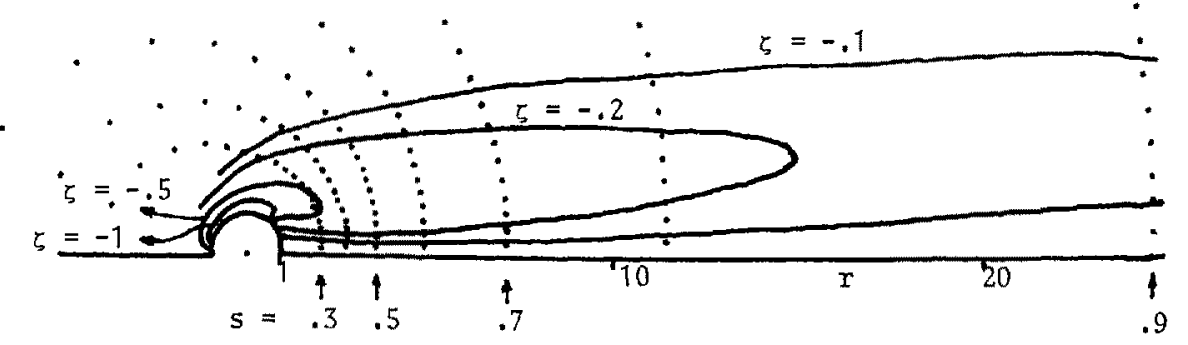

Fig.3 Streamfunction distribution for $R=20$ calculated with a $10 \times 20$ grid
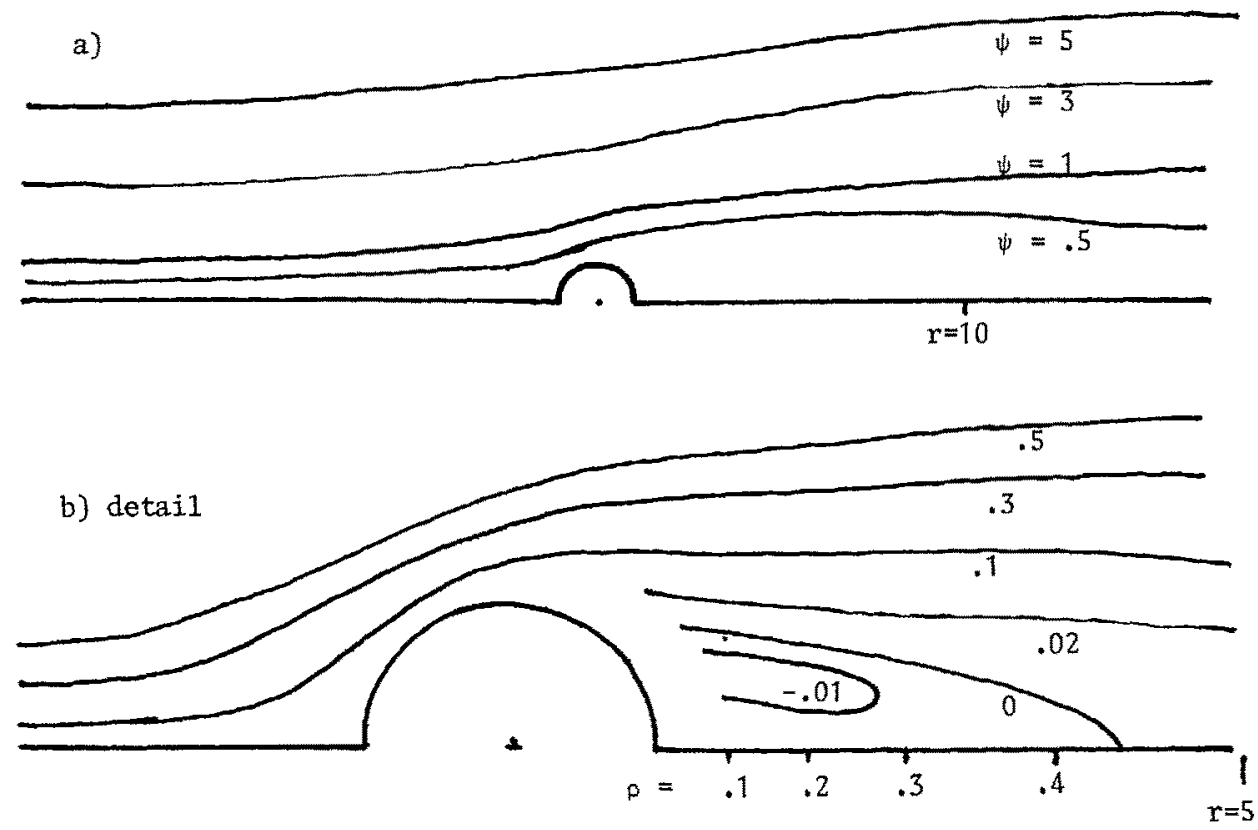